Questions vives

\section{Questions Vives}

Recherches en éducation

$n^{\circ} 21 \mid 2014$

Le travail collectif des enseignants en question(s)

\title{
Le travail collectif enseignant : allant de soi, effet de mode convenu ou analyseur décalé de la professionnalité enseignante?
}

The Collective Work of Teachers: Self-Evident, Conventional, or Useful as an

Alternative Means to Analyze the Professional Status of Teachers?

\section{Philippe Maubant}

\section{OpenEdition \\ Journals}

Édition électronique

URL : http://journals.openedition.org/questionsvives/1514

DOI : 10.4000/questionsvives.1514

ISSN : $1775-433 X$

\section{Éditeur}

Université Aix-Marseille (AMU)

Édition imprimée

Date de publication : 15 septembre 2014

ISBN : 978-2-912643-45-2

ISSN : 1635-4079

Référence électronique

Philippe Maubant, «Le travail collectif enseignant : allant de soi, effet de mode convenu ou analyseur décalé de la professionnalité enseignante? ", Questions Vives [En ligne], n² 21 | 2014, mis en ligne le 15 septembre 2014, consulté le 01 mai 2019. URL : http://journals.openedition.org/questionsvives/1514 ; DOI : 10.4000/questionsvives. 1514

Ce document a été généré automatiquement le 1 mai 2019.

\section{(†)

Questions Vives est mis à disposition selon les termes de la licence Creative Commons Attribution Pas d'Utilisation Commerciale - Pas de Modification 4.0 International. 


\section{Le travail collectif enseignant : allant de soi, effet de mode convenu ou analyseur décalé de la professionnalité enseignante?}

The Collective Work of Teachers: Self-Evident, Conventional, or Useful as an Alternative Means to Analyze the Professional Status of Teachers?

Philippe Maubant

\section{Introduction}

1 La lecture des différents textes soumis à ce numéro thématique fait état du projet de réaliser un état des lieux des recherches en cours sur la question du travail collectif enseignant. Ces travaux sont déjà très nombreux. Ils témoignent d'un projet de décrire, de saisir, de caractériser et de comprendre une forme, parmi d'autres, du travail enseignant, produit d'une double attente, celle de l'institution éducative et celle des enseignants eux-mêmes. Dans ce texte, nous proposons notre regard sur l'objet scientifique que constitue le travail collectif enseignant. Nous soumettons au lecteur aussi un ensemble de questions touchant à la fois à l'objet travaillé dans ce numéro, mais aussi aux différentes figures de la recherche portant sur le travail dans les métiers adressés à autrui. 


\section{Les cadres de référence mobilisés par les recherches ayant pour projet d'étudier le travail collectif enseignant}

2 Les travaux et écrits scientifiques antérieurs visant à fonder, problématiser et discuter ce nouvel objet scientifique que constitue le travail collectif enseignant peuvent être classés en trois catégories; une première catégorie renvoie à l'étude des pratiques enseignantes " hors de la classe ». Les textes de Lessard (2000), ceux de Marcel et Piot (2005), de Maroy (2006) ou encore l'ouvrage coordonné par Marcel, Dupriez, Périsset-Bagnoud et Tardif en 2007 cherchent à étudier, analyser et comprendre le travail enseignant hors de la classe. Cette perspective conduira des auteurs, tels Clanet et Talbot (2012) ou encore Talbot (2012) à distinguer les pratiques d'enseignement des pratiques enseignantes. Cette distinction conduit le lecteur à apprécier le travail éducatif, dans son ensemble, en intégrant, entre autres activités, l'acte d'enseigner. Elle renforce les discours institutionnels proposant de considérer d'autres tâches que celle faisant figure de cœur de métier ou de creuset de compétences (Couturier \& Chouinard, 2008). Proposer de saisir et d'analyser le travail enseignant à partir d'une conception élargie de cette activité témoigne d'une représentation du métier qui ne le réduit pas à l'acte d'enseigner, mais à un ensemble de tâches à conduire et à réaliser dans des situations « hors la classe » et qui impliquent d'autres acteurs; des collègues, des parents, voire d'autres professionnels de l'éducation, du travail social ou de la santé par exemple.

3 Ce premier cadre de référence rejoint également des travaux plus anciens considérant le travail éducatif comme un ensemble d'activités pouvant se conduire dans et hors l'école (Glasman, 1992). Ici, l'extension des frontières du travail éducatif bouleverse la conception réduite d'une activité du travail (Leplat \& Cluny, 1974). Cet élargissement du pouvoir géographique d'intervention de l'enseignant produit une prise en compte d'autres tâches, en particulier de gestion et de relation.

4 Une seconde catégorie de recherches pouvant fonder la construction de ce nouvel objet scientifique qu'est le travail collectif enseignant renvoie aux travaux portant sur les métiers-professions de l'éducation et de la formation. Ces recherches débattent in fine de la professionnalité enseignante. Décrire et caractériser le travail collectif enseignant conduit aussi les chercheurs à interroger différents objets qui n'appartiennent pas exclusivement à la sphère éducative. Citons à titre d'exemple la notion de métier (Lantheaume, 2008, Tardif \& Levasseur, 2010), celle de profession (Champy, 2010), celle d'intermétier (Cru, 1988; Mérini, 2013) ou encore celle d'interprofessionnalité (Couturier, 2002; Aubert, Manière \& Mourey, 2005). Il s'agit ici de mobiliser différentes perspectives et théories se réclamant pour la plupart de la sociologie de l'éducation et de la sociologie des professions. Avant d'étudier les transformations de l'activité du travail éducatif, il s'agit ici d'analyser les mutations du travail et plus particulièrement celles des organisations. Il y a donc ici une forte intrication entre d'une part l'analyse de l'activité du travail, l'analyse des organisations du travail et la compréhension des changements constatés dans la représentation des métiers et la prise en compte des transformations identitaires des professions et de celles des travailleurs. Ces travaux insistent tout particulièrement sur les corrélations entre trois problématiques: celle des professions, celle de l'analyse de l'activité et celle de la construction identitaire. 
5 Enfin, observer, saisir, analyser et comprendre le travail collectif des enseignants conduit aussi des chercheurs à s'inscrire dans l'étude du fonctionnement des organisations de travail. Ce troisième cadre de référence des travaux portant sur le travail collectif enseignant revendique une double paternité ; la sociologie des organisations (Mintzberg, 1990) d'une part et celle sur le partenariat éducatif d'autre part (Pelletier, 1997). Si le recours à la sociologie des organisations semble déjà acquis pour décrire le fonctionnement des établissements scolaires (Nouvelot, 1988; Derouet \& Dutercq, 1997), des recherches importantes proposent d'étudier l'établissement à la lumière de nouvelles formes de management (Bouvier, 1994; Devineau, 1998; Gather Thurler, 2000). Des travaux s'intéressent également aux capacités des organisations éducatives à répondre aux obligations de résultat (Crahay, 1994; Demailly, 1998; Lessard \& Meirieu, 2004). Enfin des recherches plus récentes proposent de repérer des traces de travail collectif au sein des organisations éducatives. Pour certaines d'entre elles, les analyses de données ont permis de donner sens à des notions comme celle de partenariat (Mérini, 2001) ou encore celles de coopération (Grangeat, 2008) ou de collaboration (Corriveau, Boyer \& Fernandez, 2011). Elles s'appuient sur l'existence d'injonctions au développement de nouvelles formes d'organisation du travail imposées par les institutions éducatives. Elles cherchent aussi, à l'instar des travaux de Letor (2009), à mettre en évidence les conditions favorables permettant aux acteurs de collaborer. Mais ces recherches font également une place importante à des formes organisationnelles de travail collectif, que l'on pourrait qualifier de spontanées, voire d'informelles. Ici se trouve posée la question de l'instigateur du travail collectif et celle de la raison d'être de ce travail. Nous soulignons ici combien l'injonction à prendre en compte le travail collectif des enseignants corrobore une autre injonction, celle de l'invitation au partenariat, à la coopération et à la collaboration. C'est ici que nous pourrions convenir que ces deux discours participent d'un effet de mode convenu sur la professionnalité enseignante, tant il reste, selon nous, à démontrer en quoi ces modalités organisationnelles du travail enseignant produisent un effet positif sur l'activité enseignante elle-même, sur l'enseignant, voire sur la classe et les élèves. Là encore, la relative faiblesse de lisibilité des intentions, des valeurs attendues et des objectifs du travail collectif pèse sur la qualité de réception de ces discours injonctifs et prescriptifs émanant de l'institution éducative.

6 Les discours sociaux sur le travail collectif des enseignants, à l'instar de ceux sur la professionnalisation des métiers adressés à autrui (Maubant, 2013), contiennent donc leur part de zones d'ombre. Nous allons tenter ici de dresser les principales questions que soulève cet objet que constitue le travail collectif des enseignants. Nous proposerons de distinguer les questions scientifiques des enjeux sociaux et professionnels.

\section{Des intentions aux valeurs supposées du travail collectif des enseignants}

7 Quel est le sens du travail collectif des enseignants? Quelle est sa raison d'être? Répondre à ces questions conduit d'abord à réfléchir aux liens de cause à effet entre le travail collectif et les processus organisationnels que ce travail collectif induit. Dès lors, il ne semble guère possible d'interroger le sens et la valeur conférés au travail collectif sans chercher à décrire et analyser les recherches portant sur les transformations des organisations éducatives. Première transformation attendue des organisations éducatives, l'invitation au développement de formes de partenariats entre l'École et 
d'autres acteurs sociaux, comme les parents bien sûr, les entreprises ou d'autres acteurs ou groupes représentant la société civile. Le développement de l'éducation à l'entrepreneuriat, par exemple, témoigne de ces invitations au partenariat entre l'École, considérée comme un monde clos qu'il convient d'ouvrir sur le monde, et des acteurs socio-économiques représentant la vie active (Houssaye, 1987). Des formes organisationnelles touchant les entreprises publiques notamment dans les pays se réclamant d'une idéologie néo-libérale se présentent sous la forme de partenariats publicprivé. Différents ouvrages portant sur cette problématique paraissent aux États-Unis et en France. Citons notamment celui de Tremblay (2003) ou encore celui de Marty, Trosa et Voisin en 2006. Ces deux ouvrages traitent des fondements des partenariats public-privé. Il est intéressant de constater combien ces modèles organisationnels reposant principalement sur des collaborations inter-organisationnelles pénètrent peu à peu les discours sur de nouvelles formes de pilotage et de gestion des organisations éducatives. Ces discours s'inscrivent principalement dans une réorganisation des institutions éducatives accueillant des élèves en processus progressif d'insertion sur le marché du travail : des écoles professionnelles ou des universités par exemple. Mais ils pénètrent aussi l'enseignement secondaire général, voire des écoles primaires. Il s'agit alors "d'éduquer à », autrement dit, initier les jeunes élèves aux fins et aux modalités de fonctionnement des acteurs sociaux et économiques.

8 Si les discours vantant les mérites des partenariats public-privé semblent rechercher une rationalisation du travail et donc une économie des coûts de production, il apparaît que les textes valorisant le partenariat en éducation soutiennent principalement une ambition d'inscrire le travail éducatif, et notamment l'apprentissage des savoirs, dans une réalité sociétale et dans une fonction d'usage social. On parie ici sur le fait qu'un ancrage de l'École dans la société donnera sens aux situations éducatives, agira sur la motivation de l'élève, participera de l'amélioration des résultats scolaires, favorisera l'insertion des jeunes sur le marché du travail. Autrement dit, le renforcement des partenariats et des autres formes de collaboration-coopération entre l'École et la société s'inscrit dans une politique d'adaptation du système éducatif aux objectifs, normes et valeurs d'un modèle économique et social. Nous pourrions ajouter que les discours politiques dressant l'apologie de partenariats entre les institutions éducatives et des organisations économiques participent sans aucun doute d'une valorisation des valeurs de l'entreprise dans un contexte de scepticisme et de doute à l'égard de modèles économiques et de modèles de management, et pour certains acteurs de l'École, dans une stratégie de résistance face à l'idéologie néo-libérale. Des auteurs tels Rastier (2013) dénonceront ces discours en considérant qu'ils constituent un signe des velléités de l'économie néo-libérale de s'approprier le marché de l'éducation et d'imposer de facto les valeurs de l'idéologie managériale.

9 Si le terme de partenariat, ou des termes équivalents visant à décrire de nouvelles configurations éducatives sont très présents dans les récentes réformes scolaires, la question du sens et des valeurs éducatives attendus de ce partenariat semble rester sans réponse. Pelletier (1997) admet combien le mot est polysémique. Comme le soulignent Mérini (2001) ou encore Boisclair et Dallaire (2008), le partenariat est d'abord et avant tout une modalité de mises en forme de relations entre différents acteurs. L'analyse de ces relations permet, selon nous, de révéler les attentes des différents protagonistes $\mathrm{du}$ partenariat. Dès lors, étudier le partenariat dans les institutions éducatives peut produire, selon nous, deux effets dans les organisations. Le premier effet est le développement de 
processus d'analyse réflexive des pratiques éducatives. Réfléchir aux partenariats créerait ainsi les conditions d'une réflexion sur les pratiques sociales et professionnelles des différents acteurs impliqués. Le second effet, celui d'interroger les discours sociaux sur le partenariat en questionnant les intentions, les objectifs explicites et implicites. L'étude de cette première transformation souhaitée des organisations éducatives, que constituent les discours sur le partenariat à l'école, révèle combien il semble essentiel d'aborder le travail collectif des enseignants en privilégiant les deux perspectives suivantes : se saisir de tels discours pour interroger la pratique enseignante; aborder les discours organisationnels en analysant leurs dimensions idéologiques, politiques, éducatives et pédagogiques. Autrement dit, considérer le travail collectif des enseignants à la lumière des idéologies et des valeurs éducatives et pédagogiques.

\section{Des différentes modalités pour penser le travail collectif des enseignants : de la coopération- collaboration à la collectivisation des pratiques enseignantes}

La seconde transformation attendue des organisations éducatives transparaît dans les discours prônant la collaboration ou la coopération. Si le travail collectif des enseignants suppose en premier lieu de créer les conditions d'un travail collaboratif ou coopératif, quel est le sens de cette collaboration ou coopération? Quelles sont les valeurs défendues dans ces mots? Dans quelles mesures le travail collaboratif ou coopératif serait-il une figure ou une forme, parmi d'autres, du travail collectif des enseignants? Quels seraient les objets de cette collaboration ou coopération? Pour répondre à ces différentes questions, il convient là encore d'identifier les trois cadres de référence mobilisés pour étudier le travail collectif des enseignants. Le premier de ces cadres porte le regard sur l'analyse de l'activité constitutive du travail éducatif, à savoir la pratique enseignante. Interroger la collaboration ou la coopération revient donc à décrire et comprendre les occasions et les conditions de mises en œuvre de processus collaboratifs ou coopératifs pensés et organisés dans et par la pratique enseignante. C'est donc ici l'analyse du travail collaboratif ou coopératif, qu'il convient d'entreprendre, en interrogeant notamment ses fondements, ce qui doit permettre de révéler les différentes conceptions de la pratique enseignante. Le texte de Thomazet et Mérini (dans ce volume), en nous proposant de définir le travail collaboratif comme une organisation en réseau de partenaires, dont l'émergence et la réussite tiennent à la capacité des acteurs à négocier et dont l'analyse fait apparaître des zones d'intermétiers, insiste sur le fait que l'étude de la coopération ou collaboration met à jour les pratiques éducatives des différents partenaires, leurs objectifs, leurs attentions et la manière dont ils pensent leur rôle, leur fonction et leur place au sein de ce réseau. Ce texte souligne combien l'analyse des processus collaboratifs ou coopératifs révèle les tensions résultant de rapports de sens, de rôles, de fonctions et de places entre les différents métiers portés par l'ensemble des partenaires. Ces tensions ou nœuds renvoient en premier lieu à des possibles divergences de sens induites par des attendus ou objectifs non explicités. Mais ils se cristallisent aussi autour de problématiques constitutives des professionnalités et des identités en jeu, notamment celle de l'expertise et celle des temporalités. En effet, collaborer ou coopérer conduit à décrire ses prérogatives, ses zones et territoires d'intervention, les spécificités de son travail et donc sa singularité professionnelle et identitaire. Décrire et énoncer son 
expertise revient à exprimer à l'autre son rôle et son pouvoir sur le réseau de partenaires. Mais une expertise se définit aussi par des temporalités. Quels sont les différents temps consacrés à la pratique enseignante et quels sont ceux prévus pour faire fonctionner le réseau de partenaires et faire vivre les processus de collaboration-coopération? En discutant des temporalités du travail coopératif ou collaboratif, on interroge de fait les temporalités du travail collectif et donc les temporalités en jeu dans la pratique enseignante. Ici encore, sans interroger le sens de ce travail collectif, il n'est guère possible de discuter, voire de négocier des temporalités de la pratique enseignante.

11 Mais il convient de ne pas se contenter d'un relevé de conceptions éducatives sur le travail éducatif, la pratique enseignante, le partenariat, la collaboration, la coopération ou encore le travail collectif des enseignants. La convocation de théories d'analyse de l'activité du travail est de toute évidence nécessaire pour prélever les traces de processus développés dans et par le travail collectif. Qu'il s'agisse de traces de collaborations, de coopérations, de négociations, ou qu'il s'agisse de repérer les contours et les formes de réseaux, de partenariats, décrire et comprendre le travail collectif des enseignants, et donc la pratique enseignante, réclame de s'intéresser aux signes pouvant décrire, expliquer et comprendre la manière dont les liens, relations ou tensions se mettent en place entre les différents acteurs.

Cette perspective nous semble prometteuse tant il convient de s'intéresser non pas seulement aux conceptions de la pratique enseignante ou aux conceptions du travail collectif, mais davantage aux interactions entre acteurs. À cet égard, le regard de la pragmatique linguistique, convoqué par la didactique professionnelle, nous rappelle, à l'instar de Piot dans le texte présent dans ce numéro, combien l'observation et l'analyse des interactions appellent "l'analyse des actes d'énonciation tels qu'ils sont utilisés par les interlocuteurs pour agir réciproquement les uns sur les autres et porte sur l'engagement des (partenaires) ${ }^{1}$ dans leur manière de dire (valeurs, intentions, normes) » (Vinatier, 2013, p. 56). Afin d'analyser et de comprendre le travail collectif, les recherches visant à analyser les interactions entre les membres du collectif nous semblent donc à privilégier. Elles permettent d'aller au-delà de la prise en compte des conceptions ou représentations sur le travail collectif et/ou sur la pratique enseignante. Néanmoins, comme le propose le texte de Germier (dans ce volume), nous ne pouvons nous passer de l'analyse des représentations du travail collectif et plus généralement, nous ne pouvons faire fi de ce que représente pour les différents acteurs la notion de collectif. Une telle analyse permettra de souligner combien les contextes et les situations interfèrent sur les représentations du travail collectif et du collectif et combien ils peuvent expliquer les raisons d'être et les fondements d'activités signifiant ce collectif. Analyser les traces de l'activité du travail éducatif comme analyser les représentations des différents acteurs impliqués dans ce travail conduit à combiner les données, à les confronter, à les faire dialoguer pour tenter de se saisir de la complexité de la pratique enseignante.

\section{Le travail collectif : analyseur de la professionnalité enseignante?}

13 Nous allons mobiliser à présent les deux autres cadres de référence convoqués par les recherches s'intéressant au travail collectif des enseignants, celui prenant appui sur la sociologie des professions et celui interpellant la sociologie des organisations. Ces deux 
cadres soulignent combien chercher à étudier les représentations du travail collectif des enseignants conduit à réinterroger la professionnalité enseignante, principalement autour de la dimension identitaire. Nous retiendrons ici trois dimensions de la problématique de l'identité professionnelle: celle d'un processus de constructiondéveloppement-reconnaissance de soi (Demazière \& Dubar, 1997), celle exprimée par la notion de porteur d'identités (Lahire, 1998), celle enfin de culture organisationnelle (Potocki Malicet, 1997). Comme le souligne Germier (dans ce volume), l'expression du travail collectif par les enseignants met en lumière leurs représentations du métier, en particulier en termes d'attentes voire d'idéal à atteindre. Dire son métier (Douard, 2003), c'est en effet exprimer des valeurs et le métier, tel qu'idéalisé, voire espéré. Prendre en compte les représentations des enseignants, c'est projeter ce que pourrait être le métier, au-delà des contingences du quotidien. Cette question se pose tant pour le praticien que pour le chercheur. La raison d'être des recherches en sciences de l'éducation d'une part et les motivations des chercheurs relevant de cette discipline d'autre part sont sans doute, elles aussi, à analyser.

La dimension des valeurs attachées à cette discipline se fait l'écho de celle constitutive du projet d'éduquer et de faire apprendre. Elle trouve son corollaire dans les valeurs assignées au métier d'enseignant. Mais ces valeurs sont-elles partagées par les chercheurs et par les enseignants? Doivent-elles l'être? Au sein d'un même groupe professionnel, sommes-nous tenus de partager la même conception de la profession? Ou convient-il d'admettre des différences de représentations, à l'intérieur d'un même groupe professionnel, a fortiori entre les différents partenaires d'une collectivité éducative ? Fautil qu'il y ait consensus sur la conception du métier, sur la représentation du travail éducatif, sur la définition du travail collectif ? Et plus généralement le fonctionnement, le développement d'une communauté requiert-il le consensus de ces membres ? Ou bien, les divergences, voire les conflits sont-ils nécessaires à la bonne santé du collectif ? Répondre à ces questions reviendrait sans doute à définir les conditions de mise en œuvre, de fonctionnement et de développement des organisations éducatives. À cet égard, il convient de rappeler que ce qui se joue dans ces débats de conceptions, dans ces confrontations de représentations, dans ces discussions de visions, c'est bien l'image du soi professionnel et sa reconnaissance par les autres. En effet, reconnaître l'autre dans l'exercice de son travail éducatif, ce n'est pas valider telle ou telle conception du métier. C'est entendre, admettre et reconnaître son alter ego comme une représentation des multiples facettes du métier d'enseignant. Et ces multiples dimensions identitaires du métier d'enseignant se développent dans et hors la classe et sur autant d'objets que suggèrent les situations éducatives (Fumat, Vincens \& Étienne, 2003) et que portent les contextes organisationnels (Barrère, 2002a). Dès lors, étudier et débattre, dans le cadre d'un collectif de travail, des conceptions de l'enseignement et de la pratique enseignante, des valeurs de l'éducation et de l'apprendre, d'un certain idéal du travail éducatif et de la profession d'enseignant, n'exige pas l'établissement d'un consensus. Il peut demeurer des nuances. Mais il est important que ce débat ait lieu, au sein de l'établissement scolaire, afin d'éviter l'illusion d'un accord partagé sur les finalités et le sens de l'éducation d'une part et sur les modalités organisationnelles et pédagogiques permettant d'atteindre ces fins d'autre part. Dès lors, le travail collectif ne serait plus seulement un signe des différentes figures de l'identité professionnelle des enseignants, mais bien davantage : un prétexte à débattre des valeurs de l'éducation. 
Décrire et comprendre les représentations du travail collectif conduit aussi à admettre le fait que chaque professionnel porte en lui plusieurs identités professionnelles qui ne sont pas exclusives les unes des autres. Chacune de ces identités puise sans doute son origine dans une lecture interprétative de la profession que chacun élabore et construit, en prenant appui sur les différentes images sociales qu'il a identifiées, qu'il a pu incorporer ou transcender, qu'il a pu réinterpréter ou rejeter. Sans nécessairement recourir aux excellents travaux visant à identifier les caractéristiques de l'évolution du métier d'enseignant (Van Zanten, 2002 ; Lessard \& Tardif, 2005; Maroy, 2006; Tardif, 2013), il demeure que l'histoire d'une profession s'incarne aussi dans les images, les propos, les métaphores, les allégories et les œuvres (Hameline, 1986). L'étude de l'histoire de la profession enseignante est donc indissociable de l'analyse de l'histoire des faits culturels comprise comme l'étude des témoignages et signes de la place conférée à l'éducation et aux professionnels de l'enseignement dans nos sociétés post-modernes. Rastier (2013) dresse un regard sans concession sur la détérioration de l'image sociétale de l'éducation, de l'école et des enseignants. Nadeau-Dubois (2013), leader du printemps érable ${ }^{2}$, considère que l'éducation ne constitue plus une priorité des gouvernements se réclamant peu ou prou de l'idéologie néo-libérale. Tout en confinant le projet d'éduquer à un simple acte marchand, le néo-libéralisme tend à magnifier le travail enseignant en l'invitant à se saisir de nouveaux espaces d'intervention. Mais ces espaces sont déjà pris. D'autres acteurs et d'autres professions prétendent aujourd'hui intervenir dans ces territoires: travailleurs sociaux, personnels de santé, animateurs, parents. Chacun tient sa place avec vigueur et tend à légitimer sa fonction sociale au regard de son champ d'intervention et de son champ d'expertise. Les enseignants sont soumis à négocier des espaces de redéfinition d'une nouvelle professionnalité incarnée par différentes identités professionnelles. Dès lors, admettre l'existence d'une pluralité d'identités professionnelles au sein d'un même métier s'inscrit bien dans une conception d'un travail éducatif à géométrie variable recouvrant des tâches hétérogènes, s'exprimant dans différents territoires sociaux et espaces professionnels, se structurant au gré de différentes temporalités, conduisant à négocier avec d'autres professions et donc d'autres professionnels et non professionnels. Tour à tour leader, animateur, accompagnateur, instructeur, évaluateur, agent de socialisation, co-éducateur, l'enseignant est bien un homme-orchestre. Mais est-il simple musicien, premier violon ou dirige-t-il l'orchestre ? Répondre à cette question suppose de prendre en compte trois problématiques : celle des attentes sociales, qui ne sont pas nécessairement identiques à celle des décideurs. Celle du choix et de l'engagement de l'enseignant dans une conception du travail éducatif au service du bien commun. Celle enfin du rapport de l'enseignant au travail éducatif et simultanément du rapport à soi. «Par son action, le soi reconnaît son pouvoir, situe son territoire, s'inscrit dans un réseau relationnel, se spécifie relativement aux autres, organise le temps. Le sujet individuel et/ou social se projette sur ce qu'il fait: gestes, techniques, production. Le produit, l'objet qu'il a conçu et fabriqué, extérieur à lui-même, porte sa marque, lui renvoie son image... » (Baillauquès, Lavoie, Chaix \& Hétu, 2002, p.43). L'enseignant ne peut penser son rôle, sa fonction et sa place, s'il n'est pas en mesure d'interroger dans une perspective dialectique son rapport au travail et son rapport à luimême. Penser l'activité du travail éducatif, les conditions de sa mise en œuvre, penser les critères de réussite de la pratique enseignante, définir les traces de l'activité d'enseignement constituent donc bien des tâches qui expriment l'image de soi et qui participent aussi d'une signification sociale que le professionnel anticipera et souhaitera communiquer aux différentes organisations dans lesquelles il agit. Adhérer ou non à la 
prescription du travail collectif conduit le professionnel à réinterroger son action et simultanément son image.

À cet égard, il pourra être intéressant d'étudier les différentes stratégies déployées par les professionnels soumis aux prescriptions du travail collectif et aux injonctions organisationnelles, que celles-ci se traduisent en discours zélateurs sur le travail collectif, en défense du travail en équipe ou encore en légitimation du travail par projet. Même si l'on peut admettre des nuances significatives entre collectif d'enseignants et équipe pédagogique, comme nous le rappelle le texte de Marcel et Murillo (dans ce volume) et celui de Piot (dans ce volume), la réussite d'une telle configuration collective dépend, non pas seulement de l'adhésion aux valeurs constitutives de ces collectifs, mais de la manière dont chaque membre de ce futur collectif saura négocier pour concilier son inscription dans des activités collaboratives ou coopératives avec la préservation de son autonomie, garante de son identité. On saura reconnaitre ici la tentation de certaines politiques éducatives désirant légitimer un discours à finalité managériale en prenant appui sur les valeurs supposées du collectif et sur les bienfaits du « vivre-ensemble » ou du «travailler ensemble », comme le souligne Périsset (dans ce volume). Face au dévoilement d'un tel discours, les professionnels développeront le plus souvent des stratégies de contournement de cette prescription, en se réfugiant derrière une posture rappelant les valeurs et les normes de la profession, telles l'autonomie du travail éducatif, la liberté académique, ou encore la spécificité du bricolage pédagogique (Meirieu, 2013).

Enfin l'identité professionnelle des enseignants, tel qu'elle peut se recomposer dans et par la prescription au travail collectif, traduit aussi les évolutions de la culture organisationnelle. Sembel (dans ce volume) considère ainsi que l'injonction au travail collectif des enseignants traduit une transformation structurelle des organisations éducatives. En réifiant le travail collectif, en cherchant à le promouvoir sans nécessairement expliciter ses intentions et ses valeurs, en contraignant par la force du groupe à adhérer à ce message du soi-disant bienfait du collectif, figure organisationnelle opposée à celle incarnée par l'individu, accablé de tous les maux, les politiques éducatives ne donnent guère le choix aux professionnels de l'éducation. Soumis à cette injonction, ils sont tenus d'adhérer à cette nouvelle doxa. Néanmoins, ils peuvent développer des stratégies de résistance, d'autant plus que l'une des caractéristiques des métiers adressés à autrui est bien cette zone d'intervention, ce cœur de métier (Couturier \& Chouinard, 2008), protégé par les textes officiels ou les conventions collectives, sorte de jardin secret préservé, derrière lequel le professionnel pourra se réfugier face à l'omniprésence du collectif. Cette forme de résistance aux prescriptions du collectif reste pour Sembel (dans ce volume) le moyen de surseoir à des formes de déqualification, voire de déprofessionnalisation. Le groupe peut exercer en effet une forme de totalitarisme. Il est aisément manipulable et versatile. La convocation de l'idée de collectif peut dès lors être considérée comme un instrument visant à pénétrer le dernier pré carré d'intervention de l'enseignant que constitue l'acte d'enseigner, en encadrant son intervention et sa pratique éducative, par des normes, des règles et valeurs imposées par le collectif. Un tel projet semble présent dès la formation professionnelle des enseignants, comme le rappelle Périsset dans son texte. Le projet idéologique et politique, semble-t-il ancien et constant, de chercher à rationaliser la pratique enseignante, traverse deux dimensions du travail éducatif.

$\mathrm{Au}$ niveau de la gestion de la classe, on observe la superposition, plus que l'articulation, de deux formes de rationalité... La relation pédagogique est à bien des égards l'objet d'un essai de formalisation experte... L'espace de la classe est aussi le 
cadre d'une autre tentative de mise en forme, basée sur l'intégration à l'espace professionnel de la gestion d'individus et de groupes très divers dans leurs comportements face à la forme scolaire et aux apprentissages. (Barrère, 2002b, p. 35)

Dans cette perspective, il conviendrait sans doute d'examiner la raison d'être de s'intéresser au travail enseignant en convoquant, parfois sans nuance et sans distance épistémologique, certaines théories d'analyse de l'activité du travail.

\section{Conclusion}

Penser collectif, faire du collectif, produire du collectif; tout semble aujourd'hui inviter les professionnels des métiers adressés à autrui, quelle que soit l'activité exercée, à s'inscrire et à s'engager avec détermination dans un collectif. Mais quelle est cette image du collectif que nous construisons? S'agit-il d'une nouvelle manière de dire le vivreensemble? Est-ce une déclinaison d'une conception communautaire de la vie sociale ? En proclamant ces discours, injonctifs, prescriptifs et normatifs, que cherche-t-on à promouvoir? La belle idée de démocratie? La victoire du groupe sur l'individu? La primauté de l'organisation sur les acteurs? Outre ces premières questions, quel sera l'objet fondateur du collectif? Une pratique en commun? Une production collective ? Un désir d'être ensemble? Ou plus prosaïquement l'image idéalisée d'un collectif? Sans aucun doute cette question du rapport entre des pratiques collectives et les images que nous construisons et que nous espérons de l'idée de collectif, se doit d'être traitée. En effet, il convient sans doute de sortir de la représentation fantasmée du collectif, y compris dans les organisations éducatives dont on sait qu'elles sont pénétrées de projets sociétaux imprégnés souvent du souffle du militantisme pédagogique. Dans un collectif se créent, se tissent et se nouent des relations humaines qui sont d'abord et avant tout des rapports de pouvoir. En contexte éducatif, ces rapports de pouvoir se développent au sein de relations d'autorité, mais aussi dans le cadre de relations hors autorité. Un collectif d'enseignants, même s'il s'inscrit dans une histoire d'expériences rassemblées, cristallise différentes formes et différentes figures de ces rapports de pouvoir. Perrenoud (1996) nous rappelle qu'il est impossible de travailler ensemble sans être confronté au pouvoir. Mobiliser ces rapports de pouvoir et inscrire la problématique du pouvoir pour penser le travail collectif des enseignants, c'est entreprendre une mise en dialogue de concepts visant à la fois à définir les sens du travail collectif, mais aussi ses modalités de mise en œuvre et de développement. Citons-en quelques-uns: collaboration et coopération, partage et échange, leadership et autorité, interactions et interrelations, symétrie et asymétrie, ego et alter ego. En situant les rapports de pouvoir au sein des débats sur le travail collectif, nous proposons d'éviter toute forme d'angélisme à l'égard des discours sur et pour le travail collectif. Nous cherchons davantage à comprendre ce que l'analyse de l'image du collectif révèle de conceptions d'une société démocratique où la question des rapports de pouvoir se pose à chaque instant. Par cette proposition, nous souhaitons interroger les deux fondements de nos sociétés démocratiques: les valeurs du bien commun d'une part ; le sens et les modalités du contrat social d'autre part. Gageons que l'analyse du travail collectif des enseignants constitue une occasion de débattre de ces fondements qui nous concernent tous. 


\section{BIBLIOGRAPHIE}

Aubert, M., Manière, D., \& Mourey, F. (2005). Interprofessionnalité en gérontologie. Paris : Érès.

Baillauquès, S., Lavoie, M., Chaix, M.-L. \& Hétu, J.-C. (2002). L'identité chez les formateurs

d'enseignants. Échanges franco-québécois. Paris : L'Harmattan.

Barrère, A. (2002a). Pourquoi les enseignants ne travaillent-ils pas en équipe? Why do teachers not work in teams? Sociologie du Travail, 44(4), 481-497.

Barrère, A. (2002b). Les enseignants au travail. Routines incertaines. Paris : L'Harmattan.

Bouvier, A. (1994). Management et projet. Paris : Hachette.

Boisclair, M., \& Dallaire, L. (2008). Les défis du partenariat dans les administrations publiques. Québec : Presses de l'Université du Québec.

Clanet, J., \& Talbot, L. (2012). Analyse des pratiques d'enseignement. Éléments de cadrage théoriques et méthodologiques. Phronesis, 1(3), 4-18.

Corriveau, L. Boyer, M., \& Fernandez, N. (2011). La collaboration intergroupe dans une école secondaire : un espace meso à s'approprier. Travail et formation en éducation. En ligne, 7 | 2010, mis en ligne le 18 février 2011, Consulté le 25 novembre 2013. URL : http://tfe.revues.org/1430

Couturier, Y. (2002). Continuité de soins et interprofessionnalité. Le rapport infirmières/ travailleuses sociales dans les activités de maintien à domicile. Recherche réalisée dans le cadre du programme du Conseil de recherches en sciences humaines du Canada.

Couturier, Y., \& Chouinard, I. (2008). La relation est-elle soluble dans la didactique ? La relationnalité dans les métiers relationnels comme objet d'une didactique des savoirs professionnels. In Y. Lenoir \& P. Pastré (Éds.). Didactique professionnelle et didactiques disciplinaires en débat (pp. 213-223). Toulouse : Octarès.

Crahay, M. (1994). (Ed.). L'évaluation et l'analyse des établissements de formation. Problématique et méthodologie. Bruxelles : De Boeck.

Cru, D. (1988). Collectif et travail de métier. In C. Dejours (Éd.), Plaisir et souffrance dans le travail (pp. 43-49). Paris : Éditions de l'AOCIP.

Demailly, L. (1998). (Éd.). Évaluer les établissements scolaires. Enjeux, expériences et débats. Paris : L'Harmattan.

Demazière, D., \& Dubar, C. (1997). Analyser les entretiens biographiques. L'exemple des récits d'insertion . Paris : Nathan.

Derouet, J.-L., \& Dutercq, Y. (1997). L'établissement scolaire : autonomie locale et service public. Paris : ESF.

Devineau, S. (1998). Les projets d'établissement. Paris : Presses universitaires de France.

Douard, O. (2003). Dire son métier. Les écrits des animateurs. Paris : L'Harmattan.

Fumat, Y., Vincens, C., \& Étienne, R. (2003). Analyser les situations éducatives. Paris : ESF

Glasman, D. (1992). L'école hors l'école. Soutien scolaire et quartier. Paris : ESF.

Gather Thurler, M. (2000). Innover au cœur des établissements scolaires. Paris : ESF. 
Grangeat, M. (2008). Coopérations et partenariats pour enseigner. Pratiques de L'éducation populaire. Créteil : SCÉREN-CRDP.

Hameline, D. (1986). L'Éducation, ses images et son propos. Paris : ESF.

Houssaye, J. (1987). École et vie active : résister ou s'adapter. Neufchâtel : Delachaux et Niestlé.

Lahire, B. (1998). L'homme pluriel. Les ressorts de l'action. Paris : Nathan.

Lantheaume, F. (2008). Tensions, ajustements, crises dans le travail enseignant : un métier en redéfinition. Pensées plurielles, 2(18), 49-56.

Leplat, J., \& Cuny, X. (1974). Les accidents du travail. Paris : Presses Universitaires de France.

Lessard, C. (2000). Évolution du métier d'enseignant et nouvelle régulation de l'éducation, Recherche et Formation, 35, 91-116.

Lessard, C., \& Meirieu, P. (2004). L'obligation de résultat en éducation. Bruxelles : De Boeck.

Lessard, C., \& Tardif, M. (2005). La profession d'enseignant aujourd'hui : évolutions, perspectives et enjeux internationaux. Bruxelles : De Boeck.

Letor, C. (2009). Comment travailler en équipe au sein des établissements scolaires? Bruxelles : De Boeck.

Marcel, J-F., \& Piot T. (Éds.), (2005). Dans la classe, hors de la classe. Évolution de l'espace professionnel des enseignants. Paris : INRP.

Marcel, J-F., Dupriez, V., Perisset Bagnoud, D., \& Tardif, M. (Éds.), (2007). Coordonner, collaborer, coopérer : de nouvelles pratiques enseignantes. Bruxelles : De Boeck.

Maroy, C. (2006). Les évolutions du travail enseignant en France et en Europe : facteurs de changement, incidences et résistances dans l'enseignement secondaire. Revue Française de Pédagogie, 155, 111-142.

Marty, F., Trosa, S., \& Voisin, A. (2006). Les partenariats public-privé. Paris : Éditions La Découverte. Maubant, P. (2013). Apprendre en situations; un analyseur de la professionnalisation dans les métiers adressés à autrui. Québec: Presses de l'Université du Québec.

Meirieu, P. (2013). Pédagogie. Des lieux communs aux concepts clés. Paris : ESF.

Mérini, C. (2001). Le partenariat en formation : de la modélisation à une application ( $2{ }^{\mathrm{e}}$ éd.). Paris :

L'Harmattan.

Mintzberg, H. (1990). Le pouvoir dans les organisations. Paris : Édition des organisations.

Nadeau-Dubois, G. (2013). Tenir tête. Québec : Lux.

Pelletier, G. (1997). Le partenariat : du discours à l'action. La revue des échanges, 4(3), 2-8.

Perrenoud, P. (1996). Pouvoir et travail en équipe. Travailler ensemble, soigner ensemble. Lausanne : Direction des soins infirmiers.

Potocki Malicet, D. (1997). Éléments de sociologie du travail et de l'organisation. Paris : Anthropos.

Rastier, F. (2013). Apprendre pour transmettre. L'éducation contre l'idéologie managériale. Paris : Presses universitaires de France.

Talbot, L. (2012). Les recherches sur les pratiques enseignantes efficaces, Questions Vives, 6(18). Accessible à : http://questionsvives.revues.org/1148

Tardif, M., \& Levasseur, L. (2010). La division du travail éducatif. Paris : Presses universitaires de France. 
Tardif, M. (2013). La condition enseignante au Québec du $19^{e}$ au $20^{e}$ siècle. Une histoire cousue de fils rouges : précarité, injustice et déclin de l'école publique. Sainte-Foy : Presses de l'Université Laval.

Tremblay, G. (2003). Les partenariats : stratégies pour une économie du savoir. Distances et savoirs, 2(1), 191-208.

Van Zanten, A. (2002). La profession enseignante en France : quelles évolutions ? Revue internationale d'éducation de Sèvres, 85-93.

Vinatier, I. (2013). Le travail de l'enseignant. Une approche par la didactique professionnelle. Bruxelles : De Boeck.

\section{NOTES}

1. Nous remplaçons les enseignants et les élèves par les partenaires dans la mesure où la citation de Vinatier renvoie aux interactions enseignants-élèves ou aux interactions enseignantsconseillers pédagogiques.

2. Grève étudiante québécoise de 2012

\section{RÉSUMÉS}

Ce texte prend appui sur l'analyse des différents articles contributifs de ce numéro. Il propose de souligner combien une réflexion sur le travail collectif des enseignants invite à interroger le sens du travail éducatif et l'enjeu de la reconfiguration des tensions entre pratiques éducatives et fonctions sociales de l'enseignement.

This text is based on the analysis of different contributing articles in this review. It proposes to emphasize reflection on the collective work of teachers invited to question the meaning of educational work and the issue of reconfiguration of the tensions between educational practices and social functions of education.

\section{INDEX}

Keywords : collective work, educational practices, partnership, professionalism Mots-clés : Collaboration, coopération, partenariat, professionnalité, travail collectif, travail éducatif

\section{AUTEUR}

\section{PHILIPPE MAUBANT}

Professeur, Université de Sherbrooke, Canada. Chercheur régulier à Institut de recherche sur les pratiques éducatives (IRPÉ), au Centre de recherche interuniversitaire sur la formation et la profession enseignante (CRIFPE-Sherbrooke), au Centre affilié universitaire (CAU) et au Centre de 
santé et des services sociaux-Institut universitaire de gériatrie (CSSS- IUGS). Chercheur associé au centre de recherche sur la formation (CRF) du CNAM Paris. 